\title{
Globular Cluster Systems in Massive Low Surface Brightness Galaxies
}

\author{
Daniela Villegas ${ }^{1,2}$, Markus Kissler-Patig ${ }^{1}$, Andrés Jordán ${ }^{1}$, \\ Paul Goudfrooij ${ }^{3}$ and Martin Zwaan ${ }^{1}$ \\ ${ }^{1}$ ESO-Garching, Karl-Schwarzschild-Str. 2, 85748 Garching b. München, Germany. \\ email: dvillega@eso.org, mkissler@eso.org, ajordan@eso.org, mzwaan@eso.org \\ ${ }^{2}$ Department of Astronomy, PUC, Vicuña Mackenna 4860, Casilla 306, Santiago 22, Chile. \\ ${ }^{3}$ STScI, 3700 San Martin Drive, Baltimore, MD 21218, USA. email: goudfroo@stsci.edu
}

Keywords. galaxies: formation, galaxies: evolution, galaxies: spiral, galaxies: star clusters

\section{Introduction}

Do LSB galaxies have globular clusters? If they do, how do the main features of these systems compare to the ones observed in other galaxies? What are the implications of these findings on the formation of the halo and the star formation history of LSB galaxies? These are the questions we are looking to address with this study.

We present preliminary results of an ACS/HST survey for globular clusters on massive LSB galaxies.

\section{Sample Description and Selection of Globular Clusters Candidates}

We selected from the catalogs of Bothun et al. (1985) and O'Neil et al. (2004) a sample of 6 LSB galaxies with masses $M \geqslant M_{M W}$, which assuming $S_{N} \sim 1$ predict between 50 and several 100 globular clusters for each galaxy. The sample was further selected to span a range of luminosity around $\mathrm{L}^{*}$, as well as about 2 magnitudes in surface brightness. Finally we applied a distance cut-off of $(m-M) \sim 33$ (i.e. $\sim 3000 \mathrm{~km} / \mathrm{s}$ ).

ACS observations of these galaxies were carried out within a single HST orbit in $g(F 475 \mathrm{~W})$ and i (F775W) bands, with exposure times of 1200s and 800s respectively. SExtractor detections of individual sources in the images were studied in order to classify them as globular clusters candidates.

Our selection criteria was based on 3 main features of the objects:

- Magnitude: $M_{g} \geqslant-10$, which is $2.5 \sigma$ above the expected turnover of the Globular Clusters Luminosity Function (GCLF) in this band, and $g<27.3$, corresponding to the completeness limit of the observations.

- Color: $0.4 \leqslant(g-i)_{0} \leqslant 2$, based on the typical color distribution of globular clusters in the MW (Harris 1996).

- Size: the half light radius of the candidates computed using KINGPHOT (Jordán et al. 2005 ) was set to $r_{i} \leqslant 10 \mathrm{pc}$ which is the upper size limit of the Galactic globular clusters (Harris 1996), and $r_{i} \geqslant 0.0125^{\prime \prime}$ that represents the point sources detection limit of our method.

\section{Results}

Figure 1 shows the color distribution of the 162 objects classified as globular cluster candidates (left panel) compared to the distribution of those Galactic globular clusters that would be detected under the same observing conditions and selection criteria we applied to the sample, if they were located in the target galaxies (right panel). Both panels show very similar distributions, 

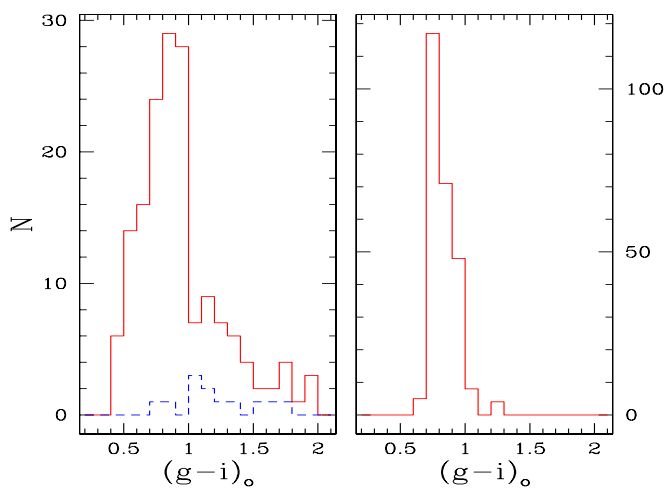

Figure 1: Left: Color Distribution of the GC candidates and the expected contamination (dashed line) compared to the distribution of the galactic globular clusters detected under the same observing conditions and selection criteria applied for the six galaxies (right).
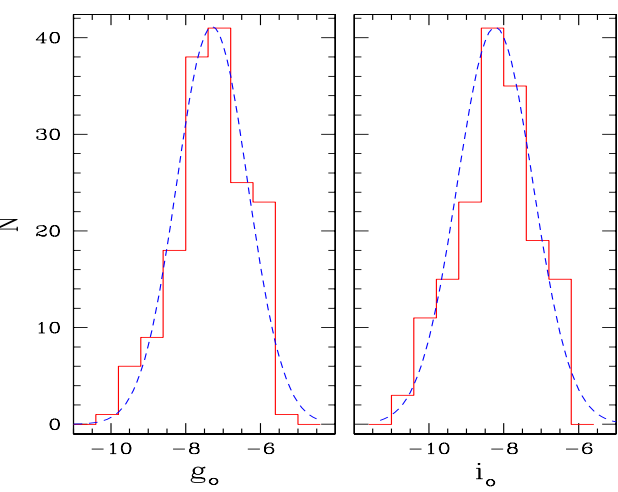

Figure 2: Luminosity distribution of the GC candidates showing the best gaussian fit for the data (dashed lines).

$\left(M_{g}, \sigma_{g}\right)=(-7.29 \pm 0.08,0.95 \pm 0.05)$

$\left(M_{i}, \sigma_{i}\right)=(-8.23 \pm 0.08,1.01 \pm 0.06)$

with a single peak at $\mathrm{g} \sim 0.8$, suggesting the presence in the LSB galaxies of a halo population similar to the one observed in our Galaxy.

The GCLF shown for both bands in Figure 2 is a good evidence for a clean sample of cluster candidates and a low contamination by young star clusters. Due to their equivalent size distribution (Scheepmaker et al. 2006) and a small overlap in color (Larsen 1999), young star clusters are likely to be the main source of contamination. Nevertheless their presence would move the GCLF to brighter magnitudes, which is not observed in this case. The dashed lines indicate the best gaussian fit to the data. These fits are consistent with the expected GCLF for a galaxy of this mass shown to be a gaussian distribution with parameters: $\left(M_{g}, \sigma_{g}\right) \sim(-7.1,1.15)$ (Jordán et al. 2006).

\section{Conclusions}

We have shown that low surface brightness galaxies do have old globular cluster populations whose general properties do not differ from those observed in normal galaxies.

The fact that the peak in the color distribution is at a position consistent with the one observed in the Milky Way indicates a coeval formation process, and the consistent profile of the GCLF tells us that further evolution of the clusters shaped their mass distribution in the same way it did in high surface brightness galaxies.

Further spectroscopic follow-up will allow to confirm the nature of the GC candidates at least in the two closest galaxies and could also provide us with new clues in order to identify the processes that make LSB galaxies different from high surface brightness galaxies nowadays.

\section{References}

Bothun, G.D., Beers, T.C., Mould, J.R. \& Huchra, J.P. 1985, AJ 90, 2487

Harris, W.E. 1996, $A J$ 112, 1487

Jordán, A. et al. 2005, ApJ 634, 1002

Jordán, A. et al. 2006, ApJ 651, 25

Larsen, S.S. 1999, A\& AS 139, 393

O’Neil, K., Bothun, G., van Driel, W. \& Monnier Ragaigne, D. 2004, A\&A 428, 823

Scheepmaker, R.A., et al. 2006, astro-ph/0605022 